\title{
Contralateral interhemispheric, transfalcine approach with asleep motor mapping for parasagittal lesions near the medial precentral gyrus
}

\author{
Michael A. Mooney, MD, and Nader Sanai, MD
}

Department of Neurosurgery, Barrow Neurological Institute, St. Joseph's Hospital and Medical Center, Phoenix, Arizona

The contralateral interhemispheric approach has several advantages for approaching parasagittal lesions, including lesions involving or approaching the medial precentral gyrus. Supplementing the interhemispheric approach with asleep motor mapping is useful for confirming the location of the corticospinal tracts from the contralateral transfalcine corridor and identifying subcortical motor fibers at the deep aspect of the resection cavity. The authors describe the contralateral interhemispheric, transfalcine approach with asleep motor mapping to resect a parasagittal metastatic lesion involving the medial precentral gyrus.

The video can be found here: https://youtu.be/L-fJ6m5kOWs.

KEYWORDS asleep; contralateral; interhemispheric; motor mapping; transfalcine; video 